\title{
"But the lithium level is normal!" SILENT Syndrome: A rare diagnosis of irreversible lithium toxicity to consider in cases of irreversible neurotoxicity
}

\author{
Sarab Sodhi*, Samantha Lee and Antoinette Spevetz \\ Temple University School of Medicine, MA Urban Bioethics, Temple University, USA
}

\section{Summary}

A case of SILENT syndrome is described in a young woman presenting with persistent altered mental status despite aggressive treatment for metabolic and infectious processes.

\section{Case}

A 47 year old female presents with altered mental status and progressive lethargy. She was noted to be at baseline mental status one day prior however witnessed "speaking gibberish" an evening at dinner prompting presentation to the emergency department.

Patient has a 30 year history of Bipolar disorder controlled with Lithium. No illicit drug use or recent changes to medication regiment. No recent illness. Surgical history of thyroidectomy for thyroid cancer, on admission, metabolic workup revealed hypernatremia, acute kidney injury and abnormal thyroid functional tests. Lithium level was $2.7 \mathrm{mEq} / \mathrm{L}$. CT-scan and Magnetic resonance imaging of the brain was negative. Work up of an infectious process was also negative including cerebral sinus fluid, blood and urine cultures. Patient was treated initially with broad spectrum antibiotics and intravenous fluids. Although serum electrolytes, thyroid function and lithium level returned to normal, she remained obtunded throughout a prolonged hospitalization. Patient was diagnosed with the Syndrome of Irreversible Lithium Induced Neurotoxicity.

\section{Discussion}

Lithium is a mood stabilizing agent used in the treatment of Bipolar disorder. It is one of the most widely studied medications and most effective agent in maintenance therapy of depression and mania while reducing the risk of short term mortality. Although efficacious, lithium is associated with a number of adverse reactions given its narrow therapeutic window. Levels should be monitored closely with a goal level of $0.5-1.3 \mathrm{mEq} / \mathrm{L}$. Clinical manifestations of toxicity including lethargy, clonus and tremor in addition to GI disturbances, abnormal thyroid and kidney functions. Evidence of toxicity can be seen with levels of (2.5 to 3.5$) \mathrm{mEq} / \mathrm{L}$ with severe central nervous manifestations when levels exceed $3.5 \mathrm{mEq} / \mathrm{L}$.

SILENT syndrome is defined as prolonged neurological complications of lithium toxicity despite removal of the medication and normalization of serum lithium levels. Lithium is known to have slow absorption into the central nervous system making serum concentrations unreliable when correlating clinically. Treatment consists of conservative management with intravenous fluids and hemodynamic support for mild to moderate cases. Hemodialysis also been used in severe toxicity. Unfortunately, neurotoxic effects of lithium can last for weeks, months or years.

\section{Conclusion}

In cases of irreversible encephalopathy without a clear etiology consider lithium toxicity for those patients on Lithium therapy even if the lithium level is normal.
Copyright: (C2016 Sodhi S. This is an open-access article distributed under the terms of the Creative Commons Attribution License, which permits unrestricted use, distribution, and reproduction in any medium, provided the original author and source are credited.
Correspondence to: Sarab Sodhi, MD, Temple University School of Medicine, MA Urban Bioethics, Temple University, USA, E-mail: sarab.sodhi11@gmail.com

Received: April 30, 2016; Accepted: May 26, 2016; Published: May 30, 2016 